Biomechanics

Elsevier Editorial System(tm) for Journal of Manuscript Draft

Manuscript Number: BM-D-16-00628R2

Title: Treatment of the Fixation Surface Improves Glenoid Prosthesis Longevity in-vitro

Article Type: Full Length Article (max 3500 words)

Keywords: glenoid; loosening; fixation failure; design; roughness; total shoulder arthroplasty

Corresponding Author: Dr. Sarah Junaid, PhD

Corresponding Author's Institution: Aston University

First Author: Sarah Junaid, PhD

Order of Authors: Sarah Junaid, PhD; Sanjay Sanghavi, MB BS; Carolyn Anglin, PhD; Anthony Bull, PhD; Roger Emery, MS, FRCS (ed); Andrew A Amis, PhD; Ulrich Hansen, PhD

Abstract: Many commercial cemented glenoid components claim superior fixation designs and increased survivability. However, both research and clinical studies have shown conflicting results and it is unclear whether these design variations do improve loosening rates. Part of the difficulty in investigating fixation failure is the inability to directly observe the fixation interface, a problem addressed in this study by using a novel experimental set-up.

Cyclic loading-displacement tests were carried out on 60 custom-made glenoid prostheses implanted into a bone substitute. Design parameters investigated included treatment of the fixation surface of the component resulting in different levels of back-surface roughness, flat-back versus curved-back, keel versus peg and more versus less conforming implants. Visually-observed failure and ASTM-recommended rim-displacements were recorded throughout testing to investigate fixation failure and if rim displacement is an appropriate measure of loosening.

Roughening the implant back ( $\mathrm{Ra}>3 \mu \mathrm{m}$ ) improved resistance to failure (P $<0.005$ ) by an order of magnitude with the rough and smooth groups failing at $8712 \pm 5584$ cycles (mean \pm SD) and $1080 \pm 1197$ cycles, respectively. All other design parameters had no statistically significant effect on the number of cycles to failure. All implants failed inferiorly and $95 \%$ (57/60) at the implant/cement interface. Rimdisplacement correlated with visually observed failure.

The most important effect was that of roughening the implant, which strengthened the polyethylene-cement interface. Rim-displacement can be used as an indicator of fixation failure, but the sensitivity was insufficient to capture subtle effects.

Level of Evidence: Basic Science Study, Biomechanical Analysis.

Copyright 2017, Elsevier. Licensed under the Creative Commons AttributionNonCommercial-NoDerivatives 4.0 International

http://creativecommons.org/licenses/by-nc-nd/4.0/ 


\section{Treatment of the Fixation Surface Improves Glenoid Prosthesis Longevity in-vitro}

2

3 Sarah Junaid $^{1,2^{*}}$, Sanjay Sanghavi ${ }^{1}$, Carolyn Anglin ${ }^{3}$, Anthony Bull ${ }^{4}$, Roger Emery ${ }^{5}$, Andrew

A Amis ${ }^{1,5}$, Ulrich Hansen ${ }^{1}$

5

6

1. Department of Mechanical Engineering, Imperial College London, SW7 2AZ, UK.

7

8

12

13

14

15

16

17

18

19

20

21

22

2. Mechanical Engineering and Design, Aston University, B4 7ET, UK.

3. Department of Civil Engineering, University of Calgary, T2N 1N4, Canada.

4. Department of Bioengineering, Royal School of Mines Building, Imperial College London, SW7 2AZ, UK.

5. Musculoskeletal Surgery, Imperial College London, London, W6 8RF, UK.

* Corresponding author: Sarah Junaid

Aston University

Mechanical Engineering and Design

Aston Triangle

Birmingham

B4 7ET, U.K.

Telephone: +44(0)121204 4177

Email: s.junaid@aston.ac.uk

22 Keywords: glenoid, loosening, fixation failure, design, roughness, total shoulder arthroplasty 


\section{Abstract}

24 Many commercial cemented glenoid components claim superior fixation designs and increased survivability. However, both research and clinical studies have shown conflicting results and it is unclear whether these design variations do improve loosening rates. Part of the difficulty in investigating fixation failure is the inability to directly observe the fixation interface, a problem addressed in this study by using a novel experimental set-up.

Cyclic loading-displacement tests were carried out on 60 custom-made glenoid prostheses implanted into a bone substitute. Design parameters investigated included treatment of the fixation surface of the component resulting in different levels of back-surface roughness, flat-back versus curved-back, keel versus peg and more versus less conforming implants. Visually-observed failure and ASTM-recommended rim-displacements were recorded throughout testing to investigate fixation failure and if rim displacement is an appropriate measure of loosening.

Roughening the implant back $\left(\mathrm{R}_{\mathrm{a}}>3 \mu \mathrm{m}\right)$ improved resistance to failure $(\mathrm{P}<0.005)$ by an order of magnitude with the rough and smooth groups failing at $8712 \pm 5584$ cycles (mean \pm SD) and $1080 \pm 1197$ cycles, respectively. All other design parameters had no statistically significant effect on the number of cycles to failure. All implants failed inferiorly and $95 \%$ $(57 / 60)$ at the implant/cement interface. Rim-displacement correlated with visually observed 41 failure.

The most important effect was that of roughening the implant, which strengthened the polyethylene-cement interface. Rim-displacement can be used as an indicator of fixation failure, but the sensitivity was insufficient to capture subtle effects.

Level of Evidence: Basic Science Study, Biomechanical Analysis. 


\section{Introduction}

2 The clinical incidence of glenoid component loosening in total shoulder replacement (TSR)

3 remains high and increases dramatically at longer follow-up. Torchia et al. (1997) reported

4 radiographic loosening rates of $44 \%$ at 9.7 years while more recent papers have reported

$548.5 \%$ at 10 years and $66.4 \%$ at 15 years (Young et al., 2011). In an attempt to lower these

6 loosening rates several types of glenoid components have been tried.

7

8 Current glenoid implant designs vary in several ways: the anchorage of the implant (keel or

9 pegs), the level of conformity between the humeral and glenoid components, curve-back 10 versus flat-back shape, and macrostructures for cement interlocking. The effect of all these 11 parameters on loosening of cemented all-polyethylene glenoid components is still not well understood.

14 One design feature that has not received much attention is the roughness of the backside of 15 the implant. Anglin et al. (2001) demonstrated a dramatic improvement to glenoid resistance 16 to mechanical loosening in two specimens after sandblasting the backside of the polyethylene 17 glenoid component. The importance of roughening the polyethylene-cement interface was also indicated by Nyffeler at al. (2003) who showed that the pull-out strengths of polyethylene pegs in cement was increased by an order of magnitude in pegs that had been roughened by sandblasting. The paper also reported clinical failure at the implant-cement 21 interface from a retrieved implant (Figures 6 and 7 in Nyffeler at al. 2003).

23 The lack of clarity of the effects of design parameters relates to the fixation being embedded 24 in the bone and impossible to observe directly. Instead, previous studies have measured 25 implant 'rim-displacement' and relied on this as an indirect measure of loosening (Anglin et 
al., 2001; Collins et al., 1992; Oosterom et al., 2004). This methodology is recommended by the American Society for Testing of Materials (ASTM F2028-14) and based on idealised cemented glenoids as a model. Due to the lack of clarity in experimental testing, studies investigating glenoid design features have largely utilised numerical methods to investigate stress patterns during various loading regimes. Lacroix and Prendergast 1997 predicted peg implants would perform better in normal bone, whereas keel would perform better in rheumatic bone, however Mansat et al. 2007 found no clear differences in stresses between peg and keel. In the case of flat-back verses curve-back, Iannotti et al. 2005 predicted higher rim displacements in flat-back designs if completely de-bonded and Swieszkowski et al. 2003 predicted high implant wear with glenoid to humeral head radial mismatch of $5 \mathrm{~mm}$ or more. Many finite element studies have been informative but cannot be predictive. Thus numerical methods can be a powerful supportive tool for the investigation of implant designs, however the variability in boundary conditions and interfacial conditions can result in varying conclusions and further highlight the need for a validated experimental approach that can be used in conjunction with numerical studies.

The aim of this study was to investigate the effect of the key design variables; keel versus peg, flat-back versus curve-back, conforming versus non-conforming and rough-backed versus smooth-backed on the life to failure of glenoid components subjected to cyclic loading. The second aim was to determine whether the ASTM recommended rimdisplacement measure would correlate with direct visual observations of failure, thus providing confidence in its use as a measure of likelihood of loosening. 
In this study, the inability to directly observe the fixation interface was solved using custommade implants (Fig. 1). These samples are clearly different from commercial glenoid prostheses but allowed direct visual inspection of the fixation for failure (Fig. 2). The samples did not vary in the third dimension and will be described as 2D models/samples. Twodimensional models are reasonable in this testing configuration because the contact in metalon-polyethylene implants is point contact where the movement is predominantly superoinferior, with relatively small anteroposterior movement (Anglin et al. 2000). This 2D methodology has also been used in a previous paper (Junaid et al., 2010).

Sixty 2D samples, including 8 different designs were manufactured based on the design of commercial implants (see Table I). No macrostructures on the keel and peg designs were included. The commercially available designs were simplified to isolate the effects of design variations without confounding the comparison by also having different macrostructures. This was carried out due to the difficulty in standardising the macrostructures, since these vary from one implant brand to another. The 60 samples were further divided into 2 groups: 24 were smooth, as machined (roughness $0-2 \mu \mathrm{m}$ ), while 36 samples were roughened to 3-5 $\mu \mathrm{m}$. Roughness measures of a typical smooth-back glenoid implant in clinical use were measured in the laboratory and found to be $1.58 \pm 0.59 \mu \mathrm{m}$ (peg) and $1.29 \pm 0.24 \mu \mathrm{m}$ (keel). A commercially available rough-backed implant was measured to have a roughness of $4.43 \pm 1.39 \mu \mathrm{m}$ (peg), thus both smooth and rough samples were within the range required. The back of the implants were sandblasted to roughen the surface including the peg and keel features and a Talysurf surface profiler (Taylor-Hobson, AMETEK Inc., Pennsylvania, USA) was used to measure the surface roughness. $R_{a}$ is most commonly used as a measure of surface roughness and is defined as the arithmetic mean of the absolute values of the roughness profile from the mean line. 
The glenoid components were implanted into a polyurethane bone substitute using PMMA bone cement (Simplex ${ }^{\mathrm{TM}} \mathrm{P}$, Stryker, New Jersey, USA) (Table II) by an orthopaedic shoulder surgeon (SS). The bone substitute blocks were prepared using a CNC machine to accurately cut out the glenoid back and accommodate a $2 \mathrm{~mm}$ cement mantle. The cement was handmixed at room temperature for approximately 90 seconds then poured into the bone substitute cavity. The volume of cement mixed was measured and consistent for each sample however, the volume used per sample was not controlled as this varied from one design to another. The implant was pressed into the cement using hand pressure and a conforming weight of approximately $0.5 \mathrm{~kg}$ was placed onto the glenoid surface to maintain the $2 \mathrm{~mm}$ mantle between the implant and bone and maintain a constant isotropic pressure during cement polymerisation. The mantle thickness was manually checked at the time of cementing to ensure it did not exceed $3 \mathrm{~mm}$ and was no less than $2 \mathrm{~mm}$.

The cylindrical humeral head (radius $24 \mathrm{~mm}$ ) was compressed into the glenoid using a horizontal load of $1800 \mathrm{~N}$ applied by a pneumatic cylinder (Junaid et al., 2010). All samples were cyclically tested with a frequency of $0.5 \mathrm{~Hz}$ under displacement-control and were tested in a water bath at $37 \pm 2{ }^{\circ} \mathrm{C}$ as described in the testing standard ASTM F2028-14 using a servohydraulic machine (Instron 8874, Illinois, USA). The tests were halted and the water

94 bath removed every 2000 cycles for the samples to be inspected visually for failure and failure progression. Subsequent to the visual inspection, a custom-made clamp fixed directly to the bone substitute block was used to clamp two linear variable displacement transducers (LVDTs) (Solatron Metrology, Bognor Regis, UK), which were aligned to measure the superior and inferior rim via reference pins inserted at the implant rim edge as specified by 
100 testing with only the LVDT removed intermittently during testing to ensure LVDT alignment and point of reference on the glenoid rim was consistent.

103 Emulating the ASTM standard, prior to cyclic testing, two additional samples of each design

104 were quasi-statically loaded in a non-destructive test to determine the load and displacement 105 to subluxation. The subluxation curves of the more- and less-conforming groups of components (Fig. 3) were averaged and $90 \%$ of the corresponding load was defined as the nominal vertical testing load during cyclic testing. The cyclic test set-up was displacement controlled to avoid sudden extensive failure progressions between inspections, and the actual

109 testing load decreased slightly as failure progressed. To ensure the $90 \%$ subluxation load was

110 maintained, the imposed displacement was readjusted every 4000 cycles. Cyclic loading was

111 carried out from the centre of the glenoid to the superior rim, as described in a previous paper

112 (Junaid et al., 2010). This imposed compressive loads on the superior rim and tensile loads on

113 the inferior rim. This loading regime is supported by clinical findings of shoulder

114 biomechanics showing predominantly superior loading and humeral head migration in vivo

115 (Bergmann et al. 2007; Trial and Nuttall 2002). Initial failure was defined as when the failure crack was first visible, mid failure as when the crack reached the keel or first peg and failure was defined as when the failure crack reached the midline of the implant (Fig. 2).

119 The Anderson-Darling test was used to test for normality of the data and a non-orthogonal 120 ANOVA test was carried out to test for statistical significance between the designs.

123 All 60 samples irrespective of design failed from the inferior edge. Fifty seven failed at the 124 implant-cement interface, two in the bone substitute and one at the cement-bone interface. 
125 Significant differences were not found between any design pairs; only roughness had a clear

126 effect $(P<0.005)$ (Fig. 4). The average number of cycles $( \pm$ SD) to failure for the rough group

127 was approximately eight times greater at $8712 \pm 5584$ compared to the smooth group at

$128 \quad 1080 \pm 1197$ cycles.

130 When using the rim displacement measure it was also not possible to identify any statistically significant differences between any of the roughened design pairs (Fig 4). Rim measurements were not originally part of the study and were not carried out on the smooth samples. An important observation was that increasing inferior rim displacement was associated with

134 progressive visual failure in all implant designs (Fig. 5 \& 6). Rim displacement greater than

$1350.61 \mathrm{~mm}$ indicated with $95 \%$ confidence if a sample was no longer intact and had experienced either mid-failure or failure.

\section{Discussion}

139 The most important finding of this study was that a rougher surface of the back of the glenoid 140 component increased the number of cycles to failure by an order of magnitude. In contrast, 141 the study did not identify any significant effects of implant design. Improving the interface 142 strength through roughness has been shown by Anglin et al. (2001), however, this is the first 143 time a study has investigated design features and roughness together.

145 The most important methodological contribution was the creation of a method to observe crack formation around an implant during cyclic loading. Previously, it has never been shown

147 that rim displacement does in fact correlate with initial or progressive loosening. The visual 148 observation of failure progression - crack growth at the implant-cement interface - was 149 correlated to the change of inferior rim displacement. The failure mode always initiated at the 
150 inferior edge of the prosthesis, along the implant-bone interface as also found in a previous study (Raiss et al., 2011).

153 The common perception that the failure is in the cement-bone interface is mostly based on

154 clinical studies that rely on radiographs. However, radiographic failure (radiolucent lines)

155 between the implant and cement interface are not visible due to polyethylene being transparent itself making it impossible to differentiate any radiolucent lines from the polyethylene. It is also important to note that radiographic loosening detects gross loosening, however this study is aimed at investigating the early signs of failure where the resolution and accuracy needed are beyond the capabilities of current radiographic images.

160

161 Therefore these clinical papers can only conclude that there are radiolucent lines in the bonecement interface but cannot conclude that there are no radiolucent lines in the implant-cement interface. Nyffeler et al. (2003) reported clinical failure at the implant-cement interface of a

164 retrieved implant. Although this failure mode appears to be rare at the stages of failure where 165 the implant is grossly loose, the authors suspect that this is an early failure mode and not as rare as is commonly thought due to the inability of radiographs to show failure in this

167 interface. Gregory et al. (2009) also found early implant-cement failure testing in cadaveric 168 bone in a similar cyclic glenoid loosening study using CT imaging, further strengthening the 169 possibility of early implant-cement failure occurring that is not identifiable clinically. Further 170 on-going work by the authors using cadaveric tissue has shown similar findings to Gregory et al. 2009 with early implant-cement interface failure evident in most samples and some cement-bone failure. 
174 The experimental loading regime for this study was used specifically to analyse the type of 175 failure mode observed and to investigate the hypothesis of whether implant-cement de176 bonding due to tensile or compressive loading is the main contributor to failure. Furthermore

177 limiting the test to superior cyclic loading is not an unreasonable testing regime as there is 178 clinical and in vivo evidence to show predominately superior loading and humeral head 179 migration in the glenohumeral joint (Bergmann et al. 2007; Trial and Nuttall 2002).

180

181 The main limitation of the study was that each design group consisted of 3 or 6 samples. The 182 reason for this discrepancy in numbers is due to the initial aim of producing 3 distinct 183 roughness groups with 3 repeats. However, the roughing techniques using sandblasting alone 184 was insufficient to achieve a higher roughness group, thus the roughness groups were pooled and compared to the smooth group. The relatively small group numbers may have prevented identifying statistical significance of design effects even when such effects may exist.

187 However, a post-hoc power analysis of the 16 groups ( 8 design groups in both the rough and 188 smooth groups) shows the study to have more than $80 \%$ power $(\alpha=0.05)$. Despite this, a post189 hoc analysis of the rough and smooth groups separately to identify the power of the design 190 features within the group found the power to be considerably less than $80 \%$. This may 191 indicate that the sample number per group was insufficient for analysing the design effects in 192 detail. Despite this, the results show a general trend that effects were not detectable within the

193 boundaries of a standardised test and therefore indicates other factors may be more influential

194 to the fixation strength such as roughness, bone quality, cement penetration, component 195 positioning and surgical technique. The number of cycles to failure in the smooth $(n=23)$ and 196 rough $(\mathrm{n}=36)$ groups demonstrated a clear dependence on roughness above all other design 197 parameters. 
199 The other limitation (and strength) is the use of the 2D sample configuration, which clearly

200 differs from the geometry of commercial glenoid components. It is difficult to evaluate the

201 effect of this simplification, but the justification is it allowed interfacial failure to be observed

202 directly and it can provide both verification and greater insight for studies using more

203 realistic models where the failure would not be visible. The loading scenario reflected a

204 higher loading regime to what is typically expected in a glenoid implant for the reason that a

2052 D experimental set up was used. In vivo work by Bergmann et al. 2011 showed peak loads

206 at $200 \%$ body weight, approximately translating to $1400 \mathrm{~N}$. Although, the compressive load

207 used at $1800 \mathrm{~N}$ was higher than the ASTM standard, it was not physiologically unreasonable

208 due to the 2D setup of the experiment. Such a set up will require a contact pressure that is

209 reflective of the physiological contact pressures (3D setup) rather than using the same loads

210 in absolute terms. Therefore to ensure comparable testing conditions, the contact pressures in

211 the 2D setup was compared to a 3D setup using Hertzian contact mechanics, showing contact

212 pressures to be comparable with 4.1 and 5.9 $\mathrm{MPa}$ in the 2D and 3D scenario respectively.

213 Therefore, while the 2D loads were higher than the clinical 3D loads, the resulting interface

214 stresses were comparable to those of the clinical setting. In addition to making allowance for

215 similar contact pressures, published in vivo data (Bergmann et al. 2011) and simulation data

216 (Anglin et al. 2000) suggests these test parameters are proximate to peak loads experienced

217 physiologically.

219 It was speculated that the omission of macrostructures may have had an effect on the results;

220 this was carried out due to the difficulty in standardising the macrostructures for

221 investigation. Since these vary from one implant brand to another, the study focussed on the

222 key design features that glenoid implants have in common. To include various

223 macrostructures could have diluted the comparison on cause of failure or resistance to failure. 
224 This study was carried out for two purposes: to investigate key design features and to validate a quantitative method of measuring failure progression. The authors plan to test this measurement method on 3D commercial implants for further validation testing. Further study on the nuances in macrostructures and their effects on fixation in keel and peg designs is needed and may be more suited to a 3D setup.

230 Large standard deviations were observed in all designs, particularly for the roughened samples, despite the controlled nature of the experiment. This may be as a result of variations in the level of cement interlocking in the implant surface and bone substitute. Indeed further study of the surface topography and surface patterns of the implant back may be interesting to investigate further but was beyond the scope of this study. In regards to the consistency of cement penetration/interdigitation, although this was not measured to ensure consistency for every implant, the cementing technique reflected clinical practice and was carried out by a clinical colleague. This is believed to more accurately reflect clinical conditions as opposed to ensuring equal cement penetration throughout the cement mantel.

There is a question of whether using bone substitute is relevant. The choice in using bone substitute that is validated to the density range and compressive properties of human bone is believed to be valid in this case and in alignment with the ASTM standard (F2028-14). By removing an element of large variation in the study this allows for more conclusive tests to be carried out on other contributing factors to fixation failure. As mentioned, the results in this study also concurs with a cadaveric study using CT imaging showing early interfacial failure at the implant-cement interface (Gregory et al. 2009) and further on-going work by the authors using cadaveric tissue also supports this finding. It may be that due to the cementing conditions, cement adheres better to dry bone substitute than real bone, however both the 
cement-bone and implant-cement interfaces experience the same load transmissions, therefore it could be argued that the failure ranking would remain the same despite possible variations in interfacial strengths. However, investigating the efficacy of different designs in light of varying bone conditions and cement penetration is still a necessary part of investigating glenoid fixation failure which is beyond the scope of this study.

This study has shown a large beneficial effect from roughening the back side of the glenoid component. Although Anglin et al. (2001) first indicated the importance of roughening, based on the immediate failure of just two smooth implants, this study provides a more comprehensive analysis. In total hip and knee replacements, implant roughness have also

259 featured in mechanical testing studies looking at the ultimate and fatigue strength of roughened metal acetabular cups and tibial trays respectively (Delaunay \& Kapandji, 1997; Miyakawa et al., 2004; Pittman et al., 2006). While there has been no other studies on the effect of roughness on glenoid fixation, in a study of tibial tray fixation Pittman et al. (2006) found that roughness increased the interface strength. The acetabular cup is not thought to fail through an eccentric load mechanism such as those for the tibial tray and glenoid component 265 (Rocking horse mechanism) however, Delauney and Kapandji (1997) did find better osseointegration of hydroxyapatite coatings on roughened cups compared to smooth. Likewise, Miyakawa et al. (2004) found osseointegration improved with roughened screws.

268 Both studies on the acetabular cup investigate cementless fixations and therefore the findings 269 from these studies cannot easily be related to the findings of this study. In the case of the 270 femoral stem, detrimental effects of increased roughness on cemented stem loosening has 271 been well documented (Howie et al., 1998) and are caused be very different fixation 272 principles than those of the glenoid component and a comparison with these studies is not 273 meaningful. 
275 Significant effects were not found between 'keel' and 'peg' designs or between flat- and curve-back. However, implanting the prosthesis into bone substitute material does not take into account that flat-backed designs require more resection of the subchondral glenoid bone 278 than curve-backed designs. Thus curve-backed designs may be more advantageous than 279 indicated in this study. Previous work evaluating commercially available implants, have 280 indicated that curve-backed and peg implants were superior to flat-backed and keel implants 281 (Anglin et al., 2001). An FE study also predicted higher rim displacement in the flat-backed models and associated this with poorer fixation (Iannotti et al., 2005) while a clinical RSA study suggested that curve-backed peg components perform better (Nuttall et al., 2007). In contrast and consistent with the results of the present study, the clinical investigation by Szabo et al. (2005) did not find a significant difference in radiolucent lines between the curve- and flat-backed glenoid implants and another study showed that intermediate clinical and radiographic outcomes are comparable between peg and keel implants (Throckmorton et al., 2010). Nho et al. (2010) and Rahme et al. (2009) also reported comparable radiographic outcomes of keel and peg implants.

This study did not find a significant effect of prosthesis conformity. Although greater conformity led to larger forces being imposed as the subluxation limit was reached, this was counterbalanced by the 'humeral head' component moving further from the centre of the 294 glenoids with less conformity, so both groups experienced loading which induced similar rocking motion. Although constrained designs have been superseded, the literature of more recent semi-constrained TSA designs is inconclusive. A recent retrieval study showed significantly longer survival of more conforming designs (5.6 years) compared to 3.1 years in 298 the non-conforming designs (Nho et al., 2010). Also, Oosterom et al. (2004) and Lacroix and 
299 Prendergast (1997) conclude that conforming designs are more durable. In contrast, Anglin et 300 al. (2001) and Orr et al. (1988) suggest that the fixation of less conforming designs is stronger 301 and Walch et al. (2002) showed a reduction of radiolucent lines associated with lower

302 conformity designs.

303

304 In this, as in other studies, the subluxation force was resisted only by the geometry of the 305 implant. In reality, the surrounding soft tissues, particularly in regard to less conforming designs, play a role in resisting subluxation. However, there is very little knowledge of the extent to which the soft tissues play a role and this greatly complicates the analysis of the effect of implant conformity. In this study, comparing the subluxation distances between more conforming and less conforming ( 3 to $4 \mathrm{~mm}$ respectively) shows a difference of $1 \mathrm{~mm}$. In absolute terms the distance is not high enough to bring large soft tissue effects and in

311 relative terms a $1 \mathrm{~mm}$ difference between conformities is small.

313 This study has established a direct link between increases in rim displacement and directly 314 observed failure in all implant designs. Although previous studies have used rim 315 displacement as an indirect measure of fixation failure (Anglin et al., 2001; Collins et al., 316 1992; Oosterom et al., 2004), there was no established link between rim displacement and 317 loosening. This association provides confidence in the use of the ASTM recommended rim 318 displacement as an indication of fixation performance.

\section{Conclusion}

321 This study highlights the importance of implant surface roughness suggesting that roughening to Ra values greater than $3 \mu \mathrm{m}$ will result in prostheses that are more likely to outlive the 323 patient and avoid revision. However, further studies are needed to investigate if the results of 
324 this in-vitro study will translate to improved performance in clinical practice. Significant

325 differences were not found in relation to fixation design features of pegs versus keel, curve-

326 versus flat-backed or conforming versus less conforming glenoid components and suggests

327 that surgeons do not need to be overly concerned about which particular fixation design to be

328 used.

329

330 The findings of the study support the use of rim displacement as a measure of fixation failure

331 and that threshold values (in the present study $0.6 \mathrm{~mm}$ ) that identifies fixation failure can be

332 established.

333

334 Acknowledgements

335 The study was funded by Arthritis Research UK. The sponsors had no involvement in the 336 design, testing, analysis, manuscript preparation and submission of this study.

338 Conflict of interest statement

339 The authors have no conflicts of interest to declare.

340

$341 \quad$ References

342 Anglin C, Wyss UP, Pichora DR. Glenohumeral Contact Forces. Proc Inst Mech Eng 343 Part H. 2000: 214 (6): 637-644. doi: 10.1243/0954411001535660

Anglin C, Wyss UP, Nyffeler RW, Gerber C. Loosening Performance of Cemented

346 Glenoid Prosthesis Design Pairs. Clin Biomech. 2001; 16 (2): 144-50. 
ASTM Standard F2028-14. Standard Test Methods for the Dynamic Evaluation of Glenoid Loosening or Disassociation. ASTM International. West Conshohocken, PA; 2008. doi 10.1520/F2028-14

Bergmann G, Graichen, F, Bender, A, Kääb, M, Rohlmann, A, Westerhoff, P (2007). In Vivo Glenohumeral Contact Forces-Measurements in the First Patient 7 Months Postoperatively. J Biomech, 40 (10): 2139-2149.

Bergmann G, Graichen, F, Bender, M, Rohlmann, A, Halder, A, Beier, A, Westerhoff, P (2011). In vivo gleno-humeral joint loads during forward flexion and abduction. J Biomech, 44 (8): 1543-1552.

359

Collins D, Tencer A, Sildles J, Matsen FA $3^{\text {rd }}$. Edge Displacement and Deformation of Glenoid Components in Response to Eccentric Loading. The Effect of Preparation of the Glenoid Bone. J Bone Joint Surg Am. 1992; 74A (4): 501-7.

Delaunay, CP \& Kapandji, AI. Acetabular Screw Rings and Surface Treatment, Clin Orth Rel Res, 1997: (340): 140-141.

Gregory T, Hansen, U, Taillieu, F, Baring, T, Brassart, N, Mutchler, C, Amis, A, Augereau, B, Emery, R. Glenoid loosening after total shoulder arthroplasty: an in vitro stems, JBJS Br, 1998: 80(4): 573-576. doi: 10.1302/0301-620X.80B4.8629 
Iannotti JP, Spencer EE, Winter U, Deffenbaugh D, Williams G. Prosthetic Positioning in Total Shoulder Arthroplasty. J Shoulder Elbow Surg. 2005;14 (1 Suppl): 111S-21S. doi: 10.1016/j.jse.2004.09.026

Junaid S, Gupta S, Sanghavi S, Anglin C, Roger E, Amis A, et al. Failure Mechanism of the All-Polyethylene Glenoid Implant. J Biomech. 2010; 43 (4): 714-9. doi: 10.1016/j.jbiomech.2009.10.019

Lacroix D, Prendergast PJ. Stress Analysis of Glenoid Component Designs for Shoulder Arthroplasty. Proc Inst Mech Eng H. 1997; 211 (6): 467-74.

Mansat, P, Briot, J, Mansat, M, Swider, P. Evaluation of the Glenoid Implant, Survival Using a Biomechanical Finite Element Analysis: Influence of the Implant Design, Bone Properties, and Loading Location. J Shoulder Elbow Surg. 2007; 16 (3S): 79S-83S. doi 10.1016/j.jse.2005.11.010

Mileti, J, Boardman III, ND, Sperling, JW, Cofield, RH, Torchia, ME, O’Driscoll, SW, Rowland, CM. Radiographic analysis of polyethylene glenoid components using modern cementing techniques. JSES, 2004:13(5):492-498. doi 10.1016/j.jse.2004.03.001

Miyakawa, S, Kawamura, H, Mishima, H, Yashumoto, J. Grit-blasted and hydroxyapatite-coated total hip arthroplasty: an 11- to 14-year follow-up study, J Orthop Sci. 2004: 9 (5): 462-467. doi 10.1007/s00776-004-0806-3 
Nho SJ, Frank RM, Verma NN, Romeo AA. Incidence of Early Development of Radiolucent Lines in Keeled Polyethylene Glenoid Components after Total Shoulder Arthroplasty. Am J Orthop. 2010; 39 (7): 333-7.

401

Nuttall D, Haines JF, Trail II. A Study of the Micromovement of Pegged and Keeled

Glenoid Components Compared Using Radiostereometric Analysis. J Shoulder Elbow

405

Nyffeler RW, Anglin C, Sheikh R, Gerber C. Influence of Peg Design and Cement Mantle Thickness on Pull-Out Strength of Glenoid Component Pegs. J Bone Joint Surg

409

410

Nyffeler, RW, Meyer, D, Sheikh, R, Koller, BJ, Gerber, C. The effect of cementing technique on structural fixation of pegged glenoid components in total shoulder arthroplasty, JSES, 2006: 15 (1): 106-111. doi 10.1016/j.jse.2005.05.002

413

414 Oosterom R, Rozing PM, Bersee HE. Effect of Glenoid Component Inclination on Its 415 Fixation and Humeral Head Subluxation in Total Shoulder Arthroplasty. Clin Biomech. 416 2004; 19 (10): 1000-8. Doi 10.1016/j.clinbiomech.2004.07.001

418 Orr TE, Carter DR, Schurman DJ. Stress Analyses of Glenoid Component Designs, Clin 419 Orthop Relat Res. 1988; 232: 217-24.

420 
Pittman, GT, Peters, CL, Hines, JL, Bachus, KN. Mechanical Bond Strength of the Cement-Tibial Component Interface in Total Knee Arthroplasty. J Arthroplasty, 2006: 21 (6): 883-888. doi 10.1016/j.arth.2005.10.006

Rahme H, Mattsson P, Wikblad L, Nowak J, Larsson S. Stability of Cemented in-Line

Pegged Glenoid Compared with Keeled Glenoid Components in Total Shoulder Arthroplasty. J Bone Joint Surg Am. 2009; 91A (8): 1965-72. doi 10.2106/JBJS.H.00938

428

Raiss P, Pape G, Kleinschmidt K, Jäger S, Sowa B, Jakubowitz E, Loew M, Bruckner T, Rickert M. Bone Cement Penetration Pattern and Primary Stability Testing in Keeled and Pegged Glenoid Components. J Shoulder Elbow Surg. 2011; 20 (5): 723-31. doi 10.1016/j.jse.2010.09.006

436

Swieszkowski, W, Bednarz P, Prendergast PJ. Contact Stresses in the Glenoid Component in Total Shoulder Arthroplasty. Proc Inst Mech Eng H. 2003; 217: 49-57. doi

437

Szabo I, Buscayret F, Edwards TB, Nemoz C, Bioleau P, Walch G. Radiographic $10.1243 / 095441103762597737$

440

Comparison of Flat-back and Convex-back Glenoid Components in Total Shoulder 441

442 Arthroplasty, J Shoulder Elbow Surg. 2005; 14 (6): 636-42. doi 10.1016/j.jse.2005.05.004

Throckmorton TW, Zarkadas PC, Sperling JW, Cofield RH. Pegged Versus Keeled 444 Glenoid Components in Total Shoulder Arthroplasty. J Shoulder Elbow Surg. 2010; 19 445 
446 Torchia ME, Cofield RH, Settergren CR. Total Shoulder Arthroplasty with the Neer

447 Prosthesis: Long-Term Results. J Shoulder Joint Surg. 1997; 6 (6): 495-505. doi $448 \quad 10.1016 / \mathrm{S} 1058-2746(97) 90081-1$

449

450 Trial, IA, Nuttall, D (2002). The Results of Shoulder Arthroplasty in Patients with 451 Rheumatoid Arthritis, J Bone Joint Surg, Br, 84B (8): 1121-1125.

452

453 Walch G, Edwards TB, Buolahia A, Bioleau P, Mole D, Adeleine P. The Influence of 454 Glenohumeral Prosthetic Mismatch on Glenoid Radiolucent Lines - Results of a 455 Multicenter Study. J Bone Joint Surg Am. 2002; 84A (12): 2186-91.

456

457 Young A, Walch G, Bioleau P, Favard L, Gohlke F, Loew M et al. A Multicentre Study 458 of the Long-Term Results of Using a Flat-Back Polyethylene Glenoid Component in 459 Shoulder Replacement for Primary Osteoarthritis. J Bone Joint Surg Br. 2011; 93B (2): $460 \quad$ 210-6. doi $10.1302 / 0301-620 X .93 B 2.25086$ 


\section{$1 \quad$ Figure and Table legends}

2

3 Fig. 1: Samples cemented into bone substitute; flat-back peg (top-left) and curve-back keel

4 (bottom-left). Two LVDTs were used to measure rim displacements at the superior and

5 inferior parts of the component (right).

6

7 Fig. 2: Failure progression showing three modes of failure; no failure, mid-failure and failure. 8

9 Fig. 3: Subluxation curve of 8 designs $(n=2$ samples each). A significant difference in

10 subluxation loads was found between the groups of implants with more or less conformity $(P$

$11=0.04)$.

13 Fig. 4: Number of cycles to failure for samples of different designs and roughness (+/- SD). 14

15 Fig. 5: Average inferior rim displacements with failure progression (before, at mid-failure and at failure respectively) for each of the eight roughened designs. Standard deviation is shown by error bars. Mid-failure: failure crack reached the keel or first peg; Failure: failure crack reached the implant mid-line.

Fig. 6: Change in rim displacement with number of cycles in the non-failed, mid-failed and

21 failed implant groups. Dashed lines are simple extensions to indicate the increase in rim-

22 displacement from the average of the originally all non-failed samples to the rimdisplacement when failure was first observed.

Table I: Overview of designs; two levels of backside roughness; two different fixations, peg and keel; two different backing shapes, flat-backed (FB) and curve-backed (CB); and two levels of conformity (larger radial mismatch indicates less conformity). 

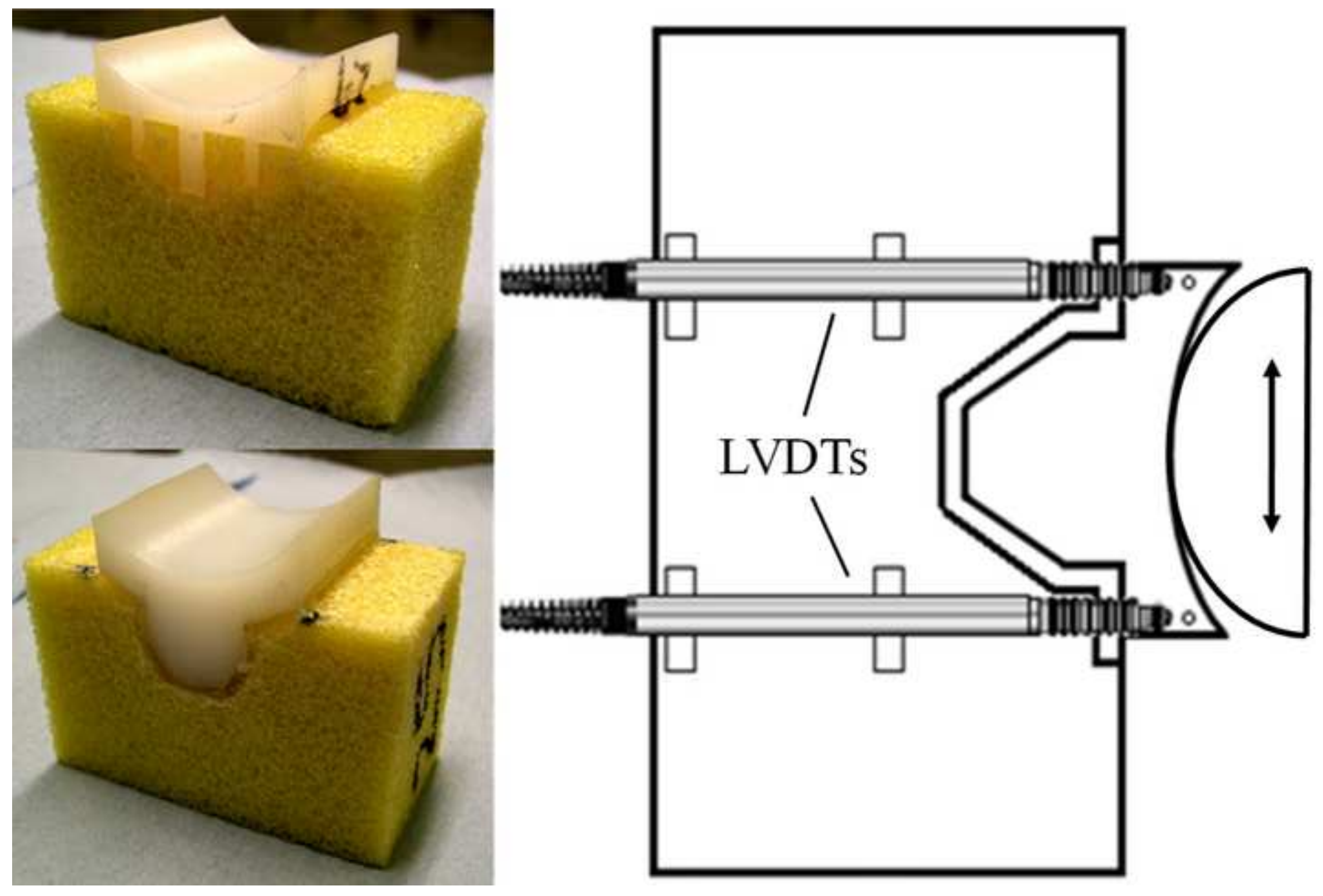


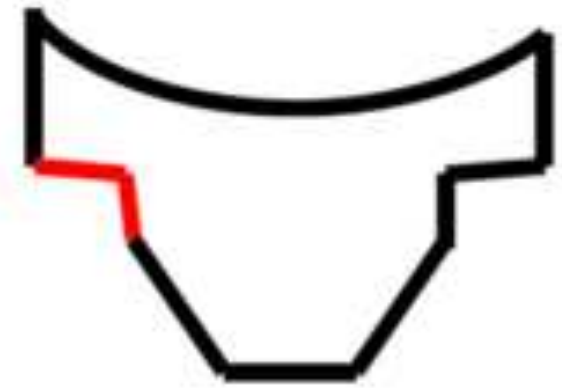

initial failure

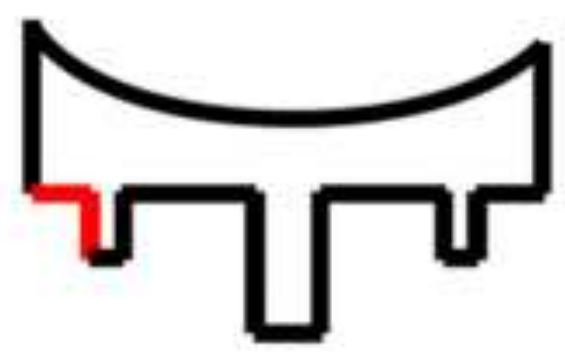

initial failure

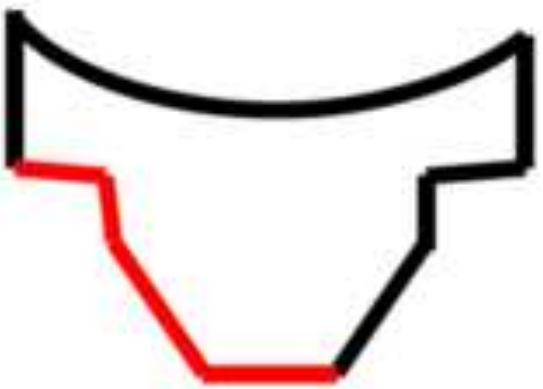

mid-failure

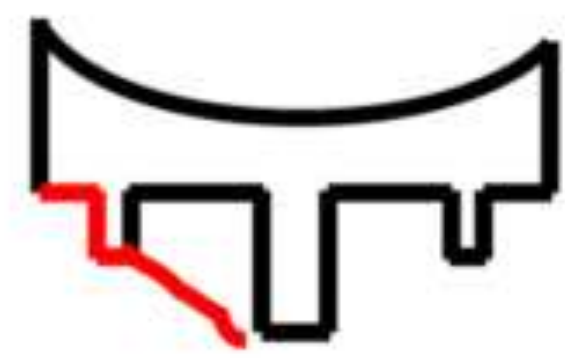

mid-failure

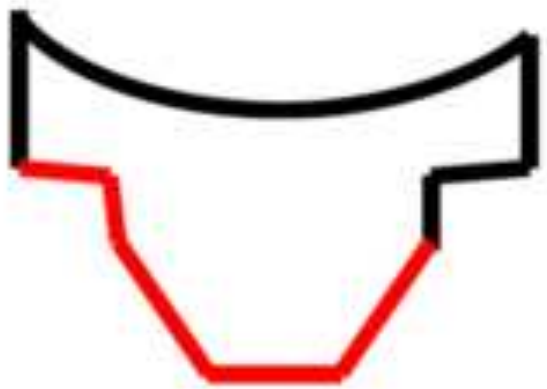

failure

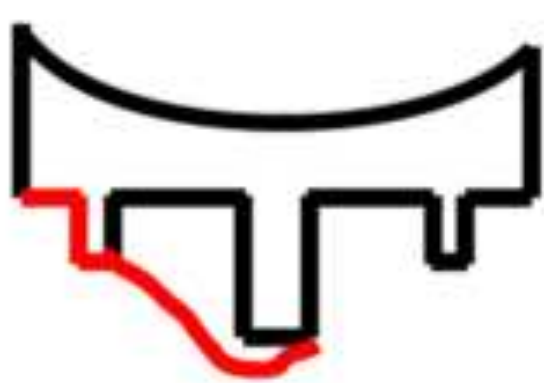

failure

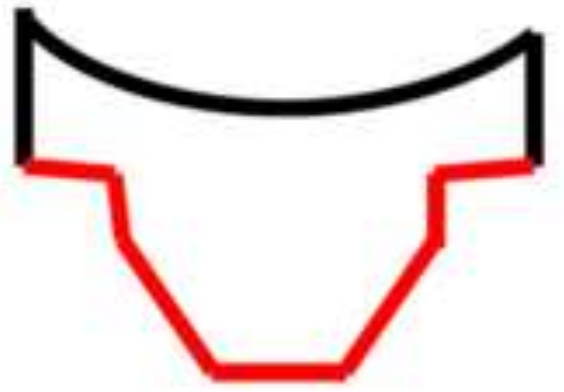

failure

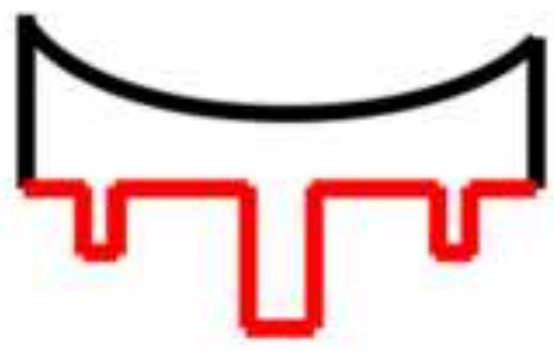

failure 
Click here to download high resolution image

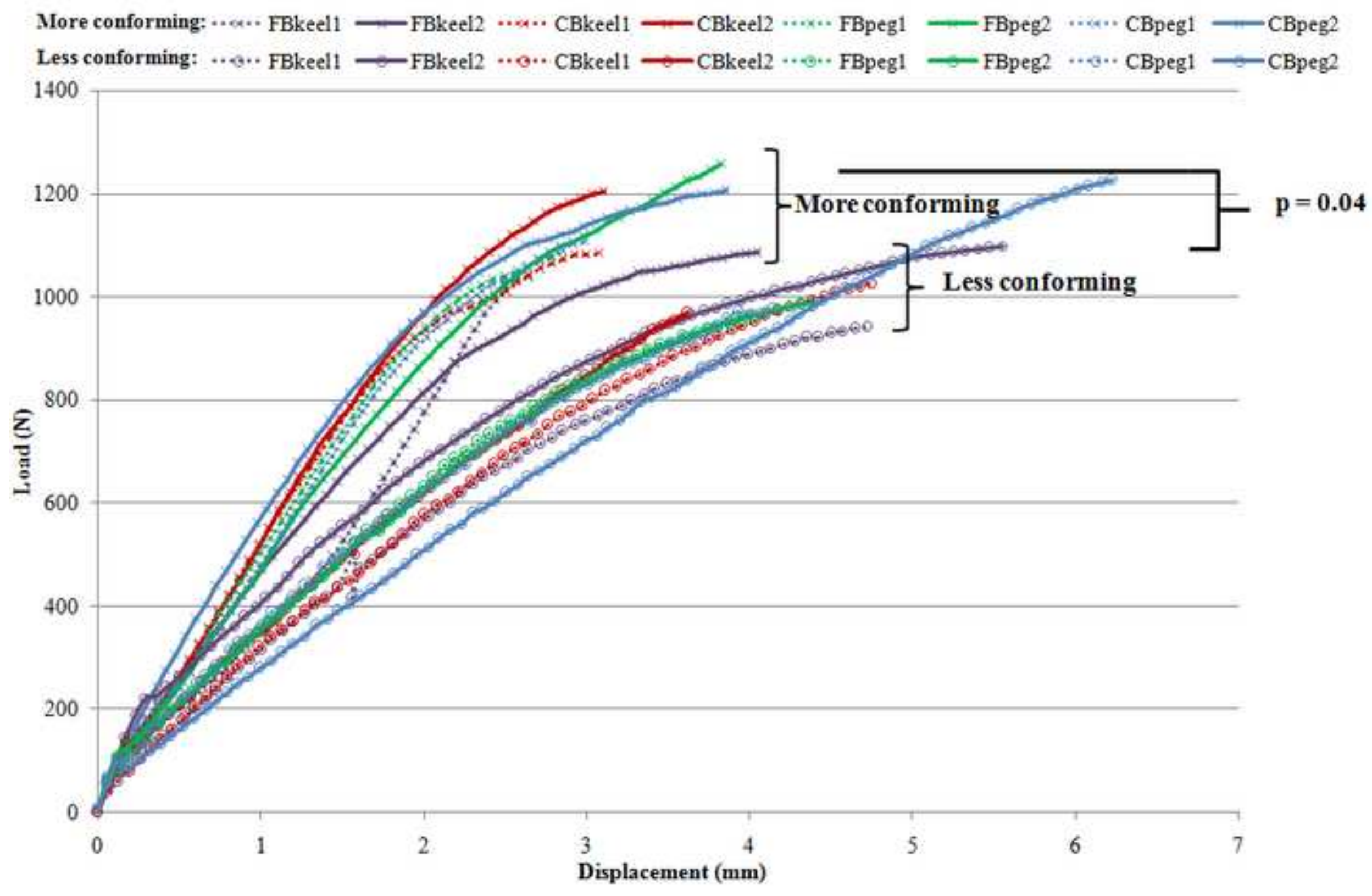


Click here to download high resolution image

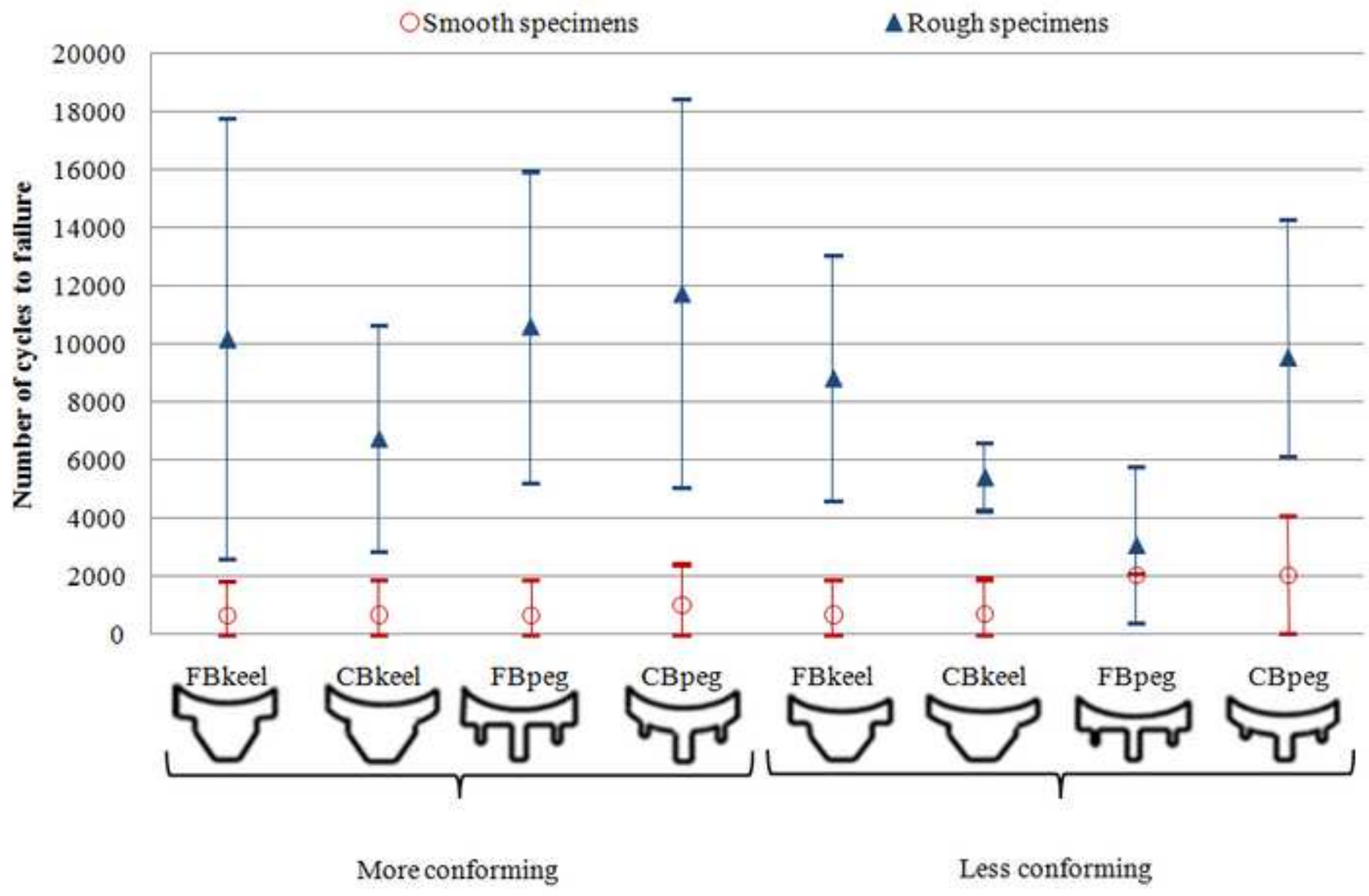




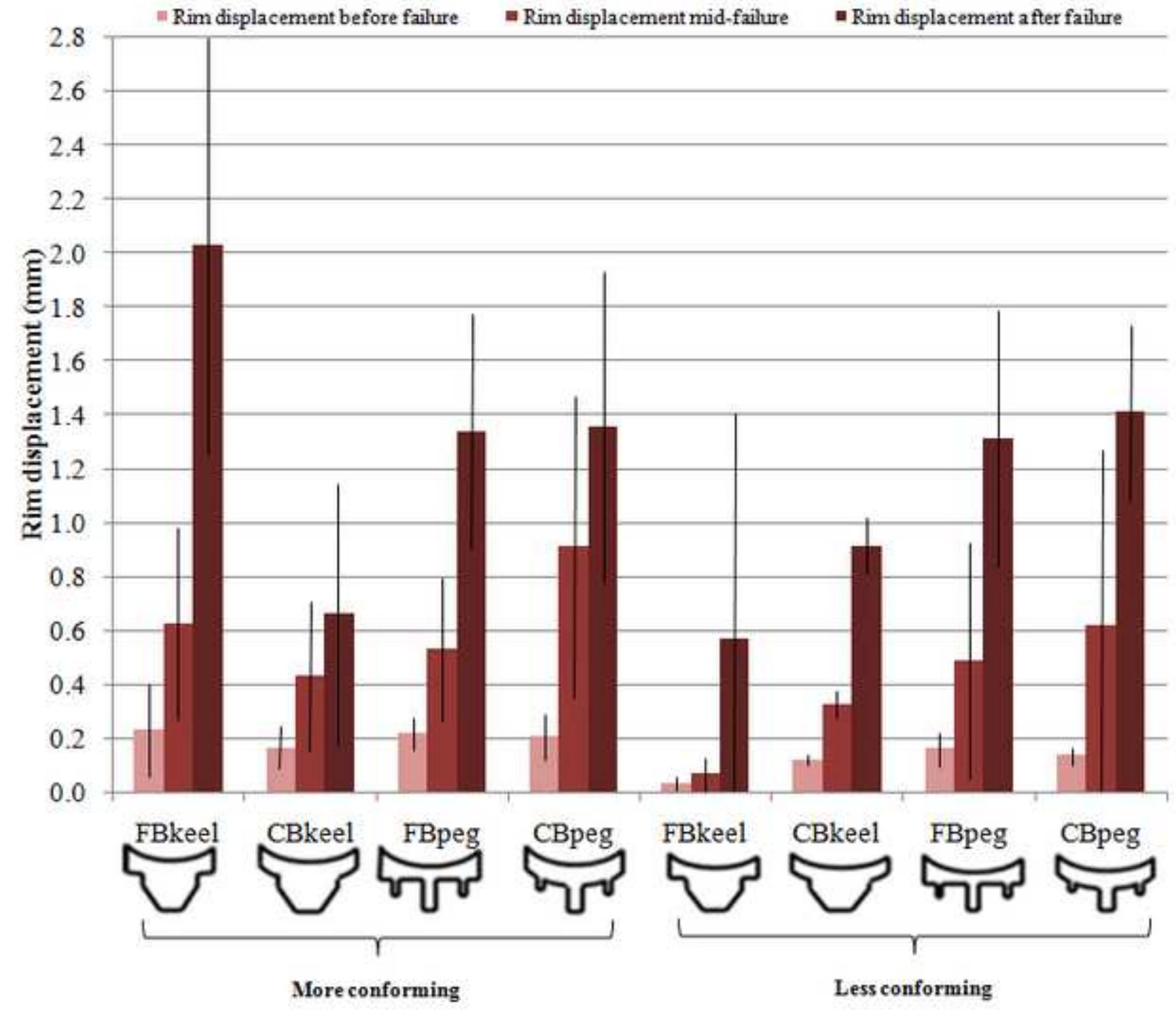

Figure 5
Click here to download high resolution image 
Change in Rim Displacement over Number of Cycles in Failed, Partially failed and Non-Failed Implants

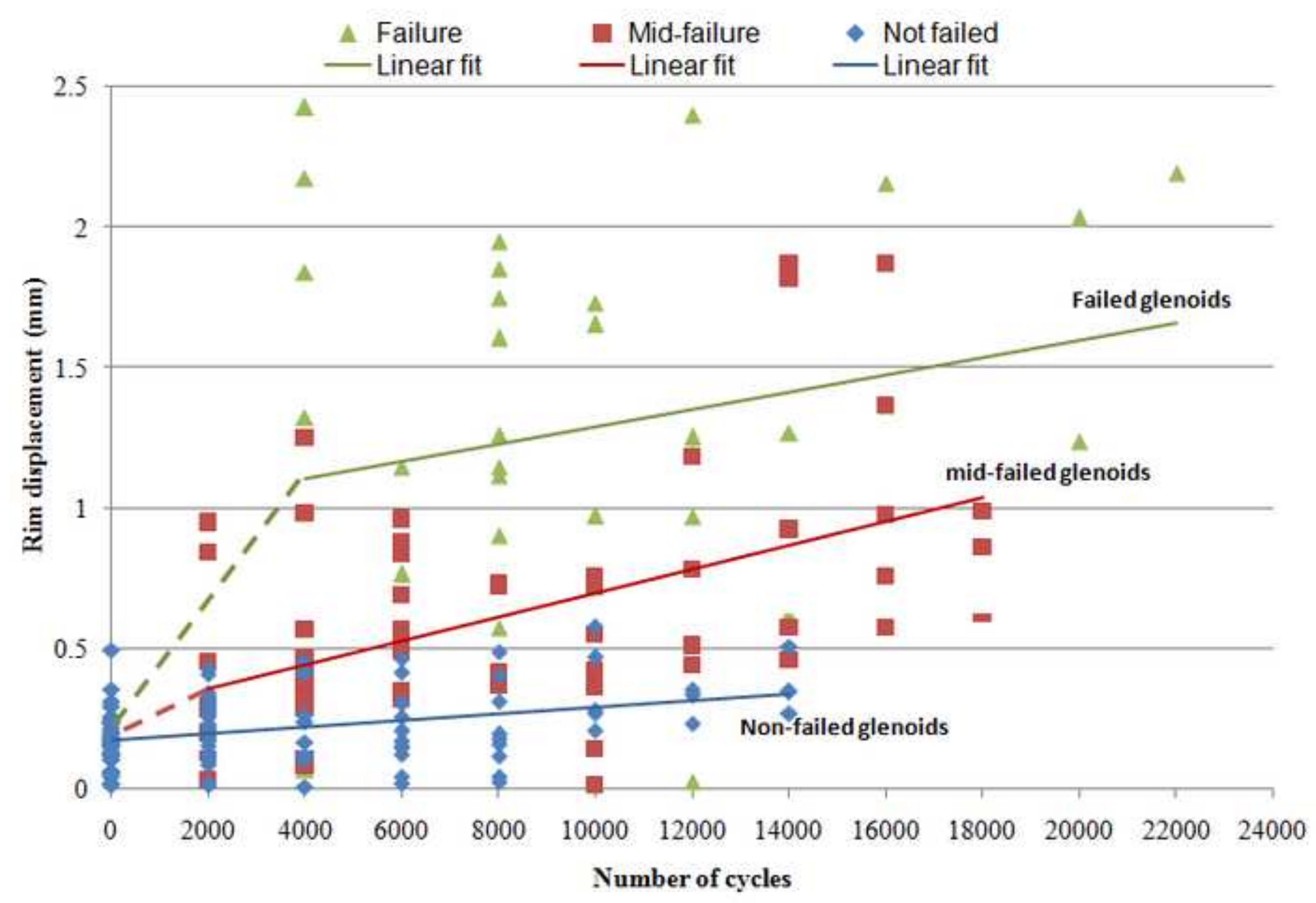




\begin{tabular}{|c|c|c|c|c|c|c|c|c|}
\hline \multirow{3}{*}{$\begin{array}{l}\text { Radial mismatch } \\
\text { (glenoid radius) }\end{array}$} & \multicolumn{4}{|c|}{ Smooth $(\mathrm{Ra}=0-2 \mu \mathrm{m})$} & \multicolumn{4}{|c|}{ Rough $(\mathrm{Ra}=3-5 \mu \mathrm{m})$} \\
\hline & \multicolumn{2}{|c|}{ Keel } & \multicolumn{2}{|c|}{ Peg } & \multicolumn{2}{|c|}{ Keel } & \multicolumn{2}{|c|}{ Peg } \\
\hline & FB & $\mathrm{CB}$ & FB & CB & FB & CB & FB & CB \\
\hline $\begin{array}{c}5 \mathrm{~mm} * \\
(29 \mathrm{~mm}) \\
\text { Less conforming }\end{array}$ & $\mathrm{n}=3$ & $\mathrm{n}=3$ & $\mathrm{n}=3$ & $n=3$ & $\mathrm{n}=3$ & $\mathrm{n}=3$ & $\mathrm{n}=3$ & $\mathrm{n}=3$ \\
\hline $\begin{array}{c}1 \mathrm{~mm} * \\
(25 \mathrm{~mm}) \\
\text { More conforming }\end{array}$ & $\mathrm{n}=3$ & $\mathrm{n}=3$ & $\mathrm{n}=3$ & $\mathrm{n}=3$ & $\mathrm{n}=6$ & $\mathrm{n}=6$ & $\mathrm{n}=6$ & $\mathrm{n}=6$ \\
\hline
\end{tabular}

* Humeral head radius was $24 \mathrm{~mm}$ leading to mismatches of $5 \mathrm{~mm}$ and $1 \mathrm{~mm}$ when related to the respective glenoid radii shown. 
${ }^{*}$ Conflict of Interest Statement

\section{Conflict of interest statement}

The authors have no conflicts of interest to declare. 